\title{
Horizontal crustal movement before the great Wenchuan earthquake obtained from GPS observations in the regional network*
}

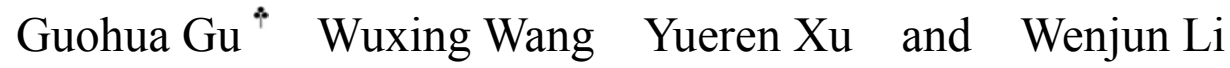 \\ Institute of Earthquake Science, China Earthquake Administration, Beijing 100036, China
}

\begin{abstract}
The great Wenchuan earthquake of M8.0 on May 12, 2008, occurred in an area with dense GPS observation stations in the regional network of the Crustal Movement Observation Network of China (CMONOC). Non-continuous observations were carried out at the 1000 GPS stations of the regional network in 1999, 2001, 2004 and 2007. The horizontal displacements at GPS stations in the regional network before the Wenchuan earthquake show that the main driving tectonic force of the earthquake was the northward pushing of the Indian plate, added at the same time by the pushing of plates on the east and south. In comparison to the displacements in other regions, the horizontal displacements near and around the seismic area is characterized by diverging eastward displacements, that is, the stations to the north of the epicenter moved in the ENE direction while those to the south of epicenter moved in ESE direction with smaller displacements at stations near the epicenter. The accuracy of the estimated strain results is briefly discussed. In order to obtain the anomalous information before the earthquake, the methods of both best fits by trend surface and statistics have been used in the study for finding the future epicentral area from the strain accumulations in the regional network observed from 1999 to 2007 before the Wenchuan earthquake. Besides the epicentral area of the western Kunlun mountain pass earthquake of $M 8.1$ in 2001, the results of best fits by trend surfaces of the strain accumulations from 1999 to 2007 in the regional network show that the Wenchuan earthquake occurred at the eastern fringe of a large area with relatively large accumulations of the first shear strains and also at the northeastern fringe of a smaller area with significant accumulated areal compressions. The statistics of the accumulations of the strain components demonstrates that they also showed anomalous distribution patterns in this area and its neighborhood with increasing accumulations of both shear strains and areal compressions.
\end{abstract}

Key words: Wenchuan earthquake; horizontal crustal movement; strain accumulation; GPS; earthquake prediction CLC number: P315.72 5 Document code: A

\section{Introduction}

Since 1999, several large earthquakes occurred in western China and its neighborhood, particularly the great Wenchuan earthquake $\left(31.0^{\circ} \mathrm{N}, 103.4^{\circ} \mathrm{E}\right)$ on 12 May 2008 caused tremendous losses. Because large amount of GPS observation data have been accumulated for these events, particularly for the great Wenchuan earthquake, which makes it possible to study the anomalous crustal movements in a large area before this event. The Crustal Movement Observation Network of China (CMONOC) includes 1000 regional GPS stations, and dense GPS stations are distributed in North China and the North-South seismic belt which includes the

\footnotetext{
* Received 17 February 2009; accepted in revised form 6 May 2009; published 10 October 2009.

^Corresponding author.e-mail: ggh@seis.ac.cn
}

epicentral area of the Wenchuan earthquake and its neighborhood. Four GPS observation campaigns were carried out in 1999, 2001 (finished before the M8.1 western Kunlun mountain pass earthquake), 2004 and 2007. Each campaign lasted about six months. Because it is rather difficult to eliminate annually periodic vertical displacements (Dong et al, 2002) in the results of GPS observation campaigns, so the vertical movements in the regional network will not be discussed. This paper focuses on the study of horizontal displacements and strain accumulations before the Wenchuan earthquake by the methods of both trend surface fits and statistics. Because GPS stations in the regional network near the epicenter are mainly located in the Sichuan basin on the east side of the earthquake fault, meanwhile the GPS stations in the mountainous area on the west side of the fault are far from the epicenter, so it is impossible to get 
the displacements near the fault on the west side of the fault and detailed strain accumulations along the fault.

Results of GPS data processing obtained from the data center of CMONOC with GAMIT/GLOBK software were used for further processing and analysis in this study (Gu et al, 2001). Different global reference frames of IGS97, IGS00, IGb00 and IGS05 were used in GPS data processing, and the horizontal displacement components in the regional reference frame (or datum definition) are obtained from solutions in the global reference frames by similarity transformation $(\mathrm{Gu}, 2006$, 2007). In this way the results in the regional reference frame are not affected by different global reference frames in general. So the crustal movements before the Wenchuan earthquake in the regional network are obtained.

\section{Horizontal displacement and strain accumulation in the regional network before the great Wenchan earthquake}

In order to get scientific explanations of regional crustal movements, stable regional reference frames (or datum definitions) have been used in regions such as Europe and North America (Blewitt, 2007). Usually both the horizontal and vertical components are considered in the regional reference frames. Because the horizontal and vertical displacements are of different fea- tures and only the solutions in the global reference frames have been available, the regional reference frames only for the horizontal displacements or only for vertical displacements have been used in this and previous studies (Gu et al, 2001, 2004).

In order to avoid the uncertainty of coseismic and post seismic displacements in the estimation of displacement velocity, horizontal displacements at about 1000 stations in the regional network, with a few stations outside China, were obtained from GPS observations from 1999 to 2007. In the regional reference frame for horizontal displacements, 98 stable stations with displacement velocities less than $2 \mathrm{~mm} / \mathrm{a}$ and most of them with velocities less than $1 \mathrm{~mm} / \mathrm{a}$ are selected; and they are located in eastern part of China and most of them are in North China. Figure 1 shows the horizontal displacements from 1999 to 2007 with the selected reference frame. The displacements in the figure exhibit rather regular features of mutual interactions and mutual constraints of different tectonic blocks or different regions. The coseismic and postseismic displacements of the M8.1 western Kunlun mountain pass earthquake in 2001 are striking in the figure with accumulated displacement of $438.1 \mathrm{~mm}$ at JB51, a GPS station $231 \mathrm{~km}$ away from the epicenter. It includes $334.5 \mathrm{~mm}$ coseismic displacement obtained from 2001 and 2004 GPS observations. Figure 1 also shows that the main driving tectonic force of the earthquake was the northward pushing from the

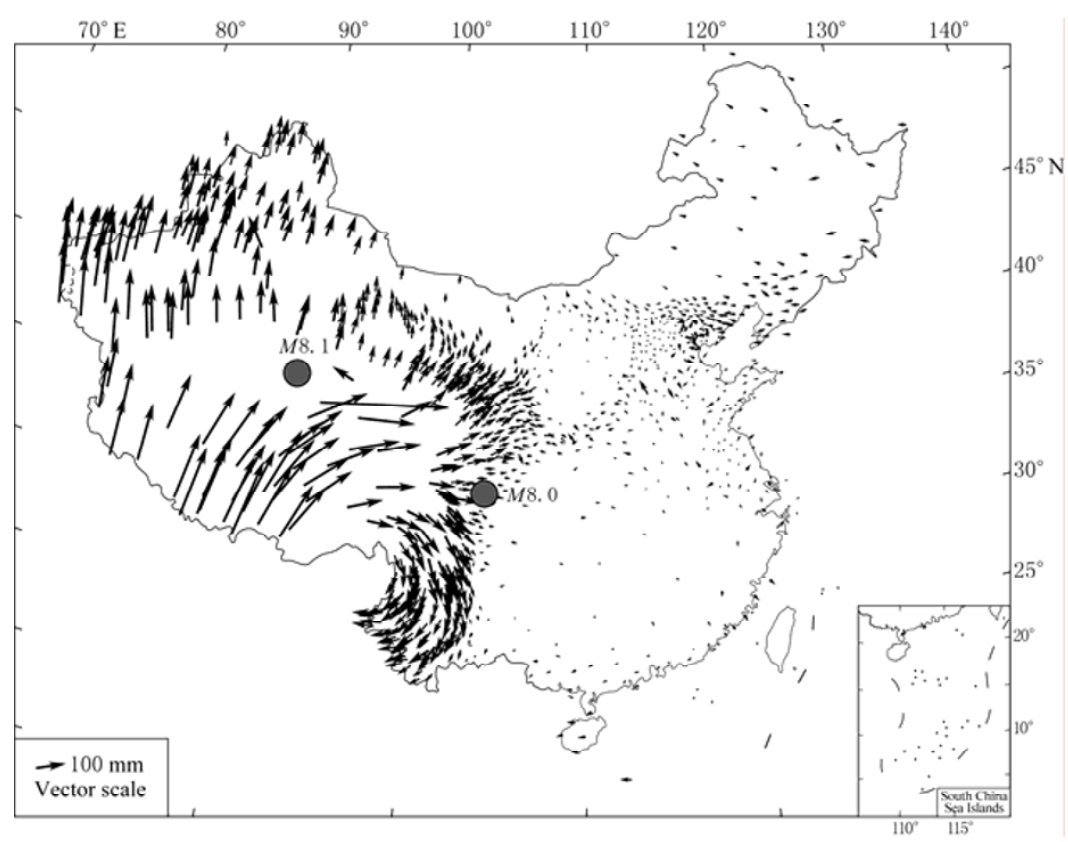

Figure 1 Horizontal displacements in Chinese mainland with reference frame in East China from 1999 to 2007. 
Indian plate, added at the same time by the pushing of plates on the east and south. In comparison to the displacements in other regions, the most striking feature of the horizontal displacements near and around the earthquake area was the diverging eastward displacements, that is, the stations to the north of the epicenter moved in the ENE direction while those to the south of epicenter moved in ESE direction with smaller displacements at stations near the epicenter. The horizontal displacements suggest that the eastward displacements near the epicenter were strongly obstructed by much more rigid block on the east.

This paper calculates the strain components on the ellipsoid from displacements at three stations for each of the automatically formed Delauney triangles. The triangles do not overlap each other (Gu et al, 1998, 2001). The strain calculation is a mathematic method regardless of fault crossing in the triangles, but the results may exhibit fault motions. Calculated strain components include: two principal strains and the azimuth of the maximum principal strain, the maximum shear strain, the first and second shear strains, areal dilatation and the spin. The first shear strain $\gamma_{1}$ is the left-lateral shear strain in $\mathrm{N} 45^{\circ} \mathrm{E}$ direction or right-lateral shear strain in $\mathrm{N} 45^{\circ} \mathrm{W}$ direction or the compression in north direction and extension in east direction; while the second shear strains $\gamma_{2}$ is the left-lateral shear strain in north direction or right-lateral shear strain in south direction or the compression in NW direction and extension in NE direction.

Though residuals in strain estimations for each triangle are 0 , the estimated strain components are still affected by the configurations of the triangles, their sizes and the errors in displacements. Besides the standard deviations in the displacements, the further the triangles deviate from the equilateral triangles and the shorter the side lengths of the triangles, the larger the standard deviations in estimated strain components. Standard deviations of the estimated strain components are affected by these factors, which could be indexes of the quality of the station configurations used in strain calculations. In strain estimation the standard deviation of the each horizontal component is assumed to be $2 \mathrm{~mm}$. It means that the standard deviation of the linear strain for a baseline of $20 \mathrm{~km}$ is $1.0 \times 10^{-7}$, then the standard deviations of strain components are estimated with the propagation of the covariance. The actual standard deviation of each horizontal displacement is probably smaller than that assumed in the estimation. For the stable blocks, such as the Ordos block, statistics of strain estimations for different periods from 1999 to 2007 (including 1999 to 2001, 2001 to 2004 and 2004 to 2007) shows that the absolute values of the first and second shear strains, the areal dilatation and the maximum shear strains are less than $1.0 \times 10^{-7}$. This statistics indicates that at least the standard deviations of these strain components are not larger than $1.0 \times 10^{-7}$. Very large first shear strains with very large standard deviations are deleted and the first shear strains larger than $2.0 \times 10^{-7}$ and also larger than their two standard deviations are deleted. Then strain components of 2002 triangles in the regional network for the period from 1999 to 2007 are obtained. Only 16 triangles mainly in North China and Yunnan of Southwest China are deleted for only a few of their strain components or absolute values are significantly large.

Results of strain estimations show that the spatial and temporal distribution of strain accumulations is inhomogeneous with complicated patterns similar to the seismic activities. They may show trend of changes in localized areas, but in general they show random features. At present it is rather difficult to model the strain fields with deterministic models. In order to predict the future earthquake areas, some approaches have been tried to find out the areas with significant strain accumulation. The western Kunlun mountain pass earthquake occurred in the regional network during the period from 2001 to 2004 and because of large coseismic and postseismic displacements, there were large coseismic strain steps and postseismic strain accumulation near the epicenter. Among the strain components, the first and second shear strains and the areal dilatation are scalar components closely related with earthquake occurrences. Analysis on these scalar components during this period illustrates that when the strain components are fitted by the trend surfaces compiled by GMT mapping tool, the colored maps of strain component distribution show, in a more direct and attractive way, the epicentral area and its neighborhood with the most significant strain accumulation. The results by this method agree in general with those from contour maps. Based on this study strains in the regional network before the Wenchuan earthquake were analyzed.

Figure 2 shows the distribution of the first shear strain $\left(\gamma_{1}\right)$ accumulation from 1999 to 2007 obtained by trend surface fitting. In order to get a more striking picture before the Wenchuan earthquake without affecting the analysis result, the strains larger than $1.0 \times 10^{-6}$ resulted mainly from the western Kunlun mountain pass 
earthquake were deleted in the fitting. The area with the most significant strain accumulation was on the west side of the Wenchuan earthquake. Though large accumulated strains were deleted, the effects of coseismic and postseismic deformation near the epicenter of the western Kunlun mountain pass earthquake can still be seen in the figure. On the west side of the Wenchuan earthquake, the accumulated first shear strain was left lateral shear strain in $\mathrm{N}^{\circ} 5^{\circ} \mathrm{E}$ direction with the largest accumulated strain about $-4.0 \times 10^{-7}$ as detected from the trend surface fitting. An anomalous shear strain belt was in general along the Xianshuihe fault, and the other was generally parallel to the earthquake fault. It should be noted that the results of trend surface fittings only indicate the dominance of the first shear strains in $\mathrm{N} 45^{\circ} \mathrm{E}$ direction in the anomalous area. This fact is in good agreement with the discrete results of triangles. Figure 3 shows distribution of the fitted dilatation $(\Delta)$ accumulation near the epicenter from 1999 to 2007 . It can be shown that the Wenchuan earthquake occurred at the eastern fringe of a smaller area with relatively large accumulated compression within the anomalous area in Figure 2, with the largest accumulated compression about $-3.5 \times 10^{-7}$. The results of strain estimation in the

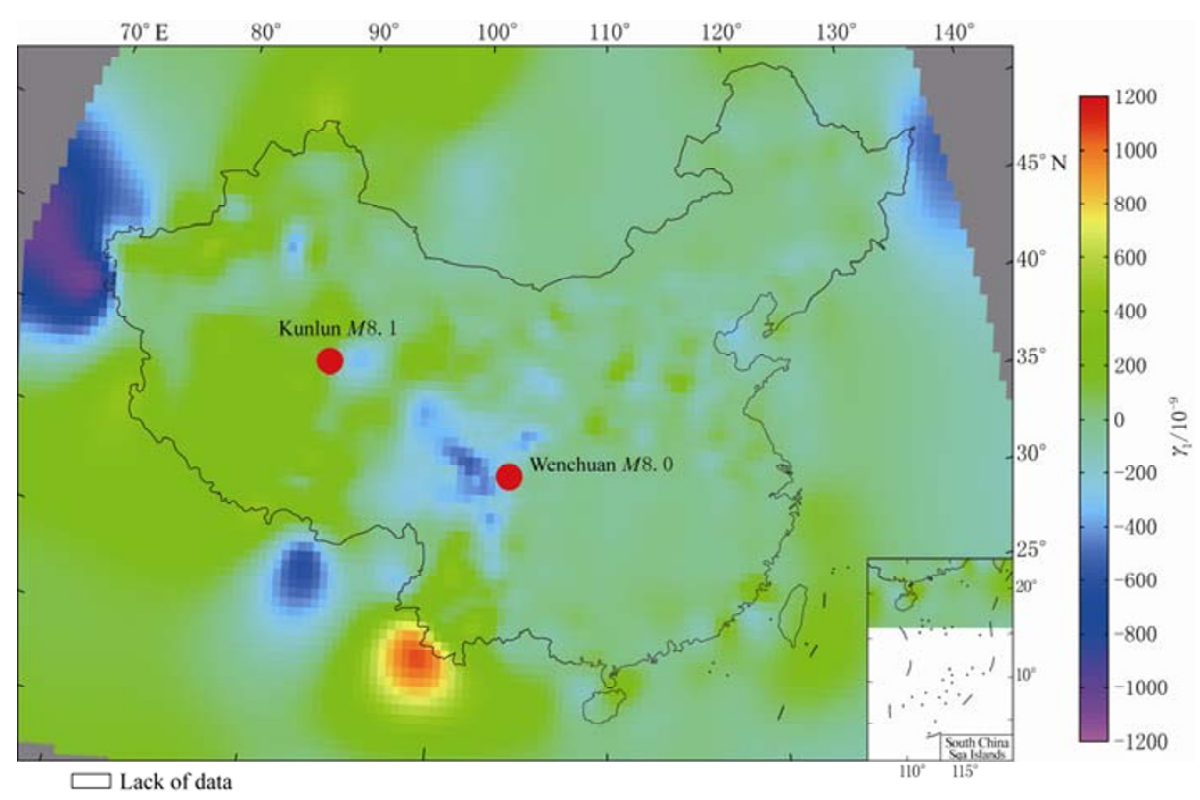

Figure 2 Accumulation of the first shear strains $\gamma_{1}$ from 1999 to 2007.

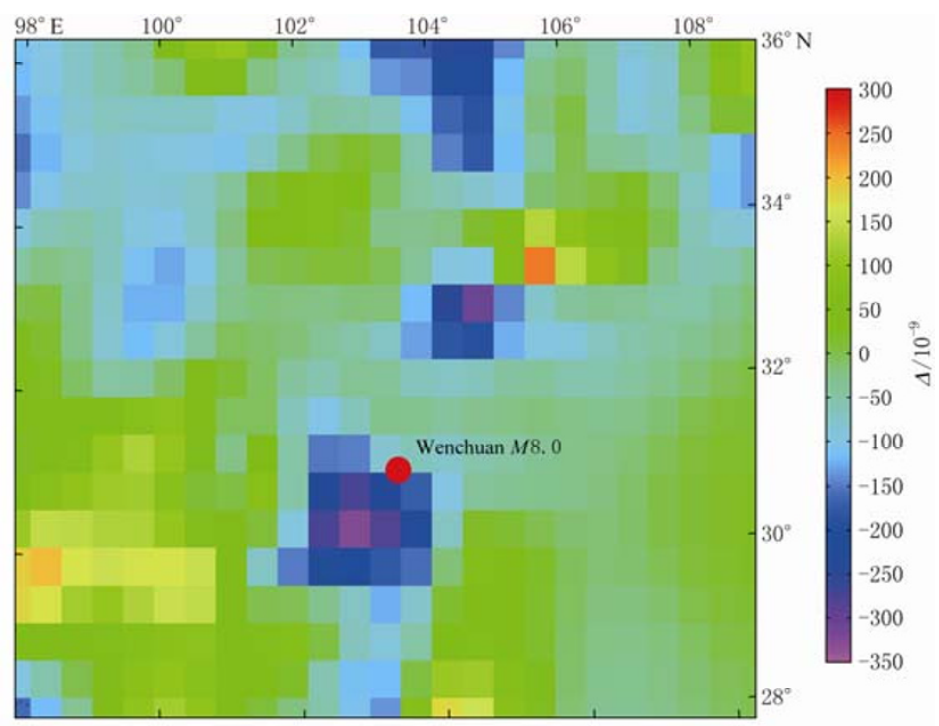

Figure 3 Accumulation of areal dilatation $\Delta$ from 1999 to 2007. 
regional network in the periods from 1999 to 2001, 2001 to 2004 and 2004 to 2007 show that the area and magnitude of the anomalous first shear strains increased gradually. Results from trend surface fittings with grid intervals of $1^{\circ}$ and $30^{\prime \prime}$ are in general the same.

Because the trend surface fittings in the areas near the border of Chinese mainland are affected by small number of triangles used in data fittings, only the results inside Chinese mainland are considered in the analysis.

The same method was used to analyze the strain accumulation in the regional network from 1999 to 2001, but no similar significant strain accumulations near the epicenter and its neighborhood before the $M 8.1$ western Kunlun mountain pass earthquake were found. The reasons could be the small number of GPS stations in the earthquake area, short time interval between two GPS observation campaigns, smaller strain accumulation and type difference of the earthquakes.

The strain accumulation observed by GPS may be results of the strain energy releasing or strain energy accumulation, including strain energy accumulation before the earthquakes. In view of similar complexity of both the seismic activities and the strain accumulation in space and time, statistic method was used to analyze the tempo-spatial patterns of strain accumulation before and after the western Kunlun mountain pass earthquake in 2001 based on GPS observations in the basic network of CMONOC (Gu and Wang, 2006). The statistic method is used again in this paper to study the strain accumulation from 1999 to 2007 in the regional network.

Statistics of the strain estimation in the regional network for the periods from 1999 to 2001, 2001 to 2004 and 2004 to 2007 shows that the distribution patterns of some components for different periods are of similar normal distribution: the distribution of the first and second shear strains, areal dilatation are almost of the same normal distribution and the maximum shear strains are of the Poisson distribution. In Figures 4 and 5, abscissa represents the number of the strain components in their ascending order, the same number does not mean that the components are from the same triangle. Statistics is made on strain components of 217 triangles in an area bounded by longitudes $98^{\circ} \mathrm{E}$ and $105^{\circ} \mathrm{E}$ and by latitudes $28^{\circ} \mathrm{N}$ and $36^{\circ} \mathrm{N}$. The area includes the anomalous area shown in Figures 2 and 3 and also includes the area of earthquake preparation, which is much larger than the aftershock area. In order to illustrate the variations nearest to the moment of the earthquake occurrence, Figure 5 shows the statistics of the first and second shear strains, areal dilatation and the maximum shear strain from 2004 to 2007. In the statistics azimuth of the maximum shear strain is not considered. In the figure the distribution of larger absolute values of the first and second shear strains, areal dilatation is significantly different from those shown in Figure 4. The maximum shear strain gives the same phenomenon. No variations that are as significant as shown in Figure 4 could be seen from statistics on the strain components from the triangles in the whole network (2 021 triangles), North China (392 triangles), Yunnan (180 triangles) and West China (229 triangles) during the same period of 2004 to 2007. Figure 5 shows that in the area with anomalous strain accumulation, the accumulated strain were large (with their absolute values of $3.0 \times 10^{-7}$ ) and the number of triangles of the same or nearly the same strain accumulation were more than that in other regions or during other periods. In comparison to the period from 1999 to 2001, the number of triangles with maximum shear strains less than $1.0 \times 10^{-7}$ decreased from 72 to 50 in the period from 2004 to 2007 .

The relationship between earthquake magnitude $M$ and their frequency $N$ can be expressed by $\lg N=a-b M$, where $a$ and $b$ are coefficients. Similar statistics of the maximum shear strains could be made, but their number is too small as compared with the number of earthquakes. In order to make direct and detailed comparison, maps of annual distribution of earthquakes with $M \geq 1.0$ in Chinese mainland in ascending order of their magnitudes from 2004 to 2008 have been obtained. Their distributions in these maps are quite similar to that of the maximum shear strain accumulation in Figure 4. Though earthquakes did not occur at fixed places and the strains observed by GPS observations at determined time intervals are obtained at fixed places, both of them are the results of crustal deformations, therefore they should be governed by similar statistic distribution.

In order to confirm the anomalies in strain accumulation around the epicenter of the Wenchuan earthquake, statistics were made on the distribution of accumulation of the first and second shear strains, areal dilatation and the maximum shear strain in different intervals for the whole network, East China, North China, the Wenchuan earthquake area, Yunnan and West China. The interval length is $1.0 \times 10^{-7}$ and the ratios of the number of triangles with strain components in each interval to the total number of triangles in a region are obtained. The number of triangles for the whole network or for the same region remains in general the same from 

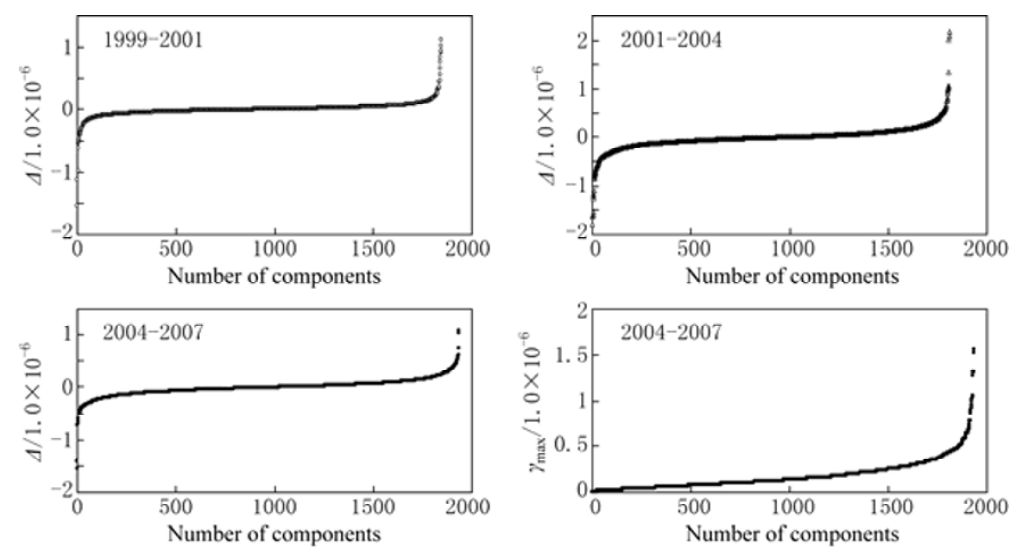

Figure 4 Distribution of areal dilatation ( $\Delta$ ) accumulation from 1999 to 2007 and distribution of the maximum shear strain $\left(\gamma_{\max }\right)$ accumulation from 2004 to 2007.
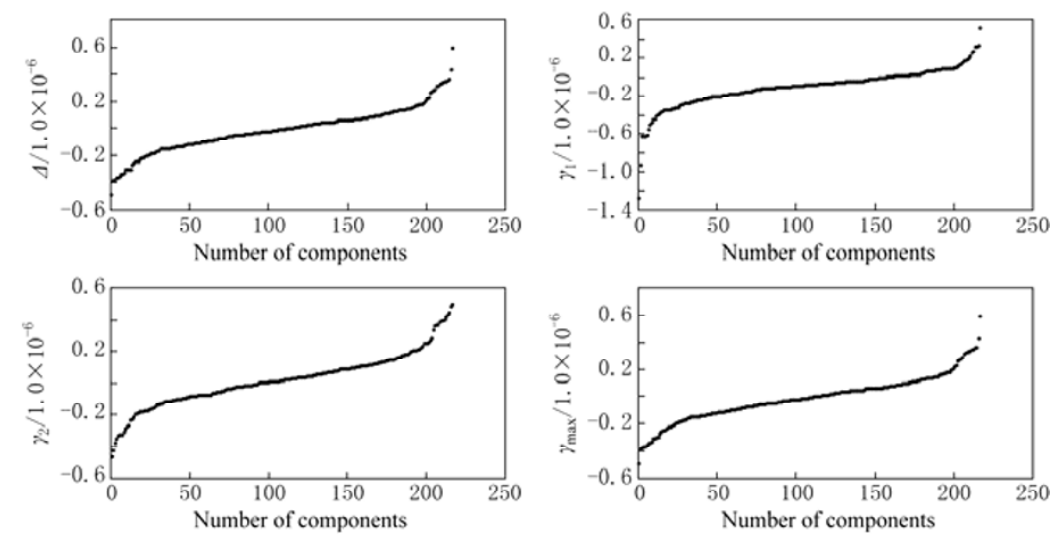

Figure 5 Distribution of accumulation of the first $\left(\gamma_{1}\right)$ and second $\left(\gamma_{2}\right)$ shear strains, the areal dilatation $(\Delta)$ and the maximum shear strain $\left(\gamma_{\max }\right)$ from 2004 to 2007 in the anomalous area in Figure 2.
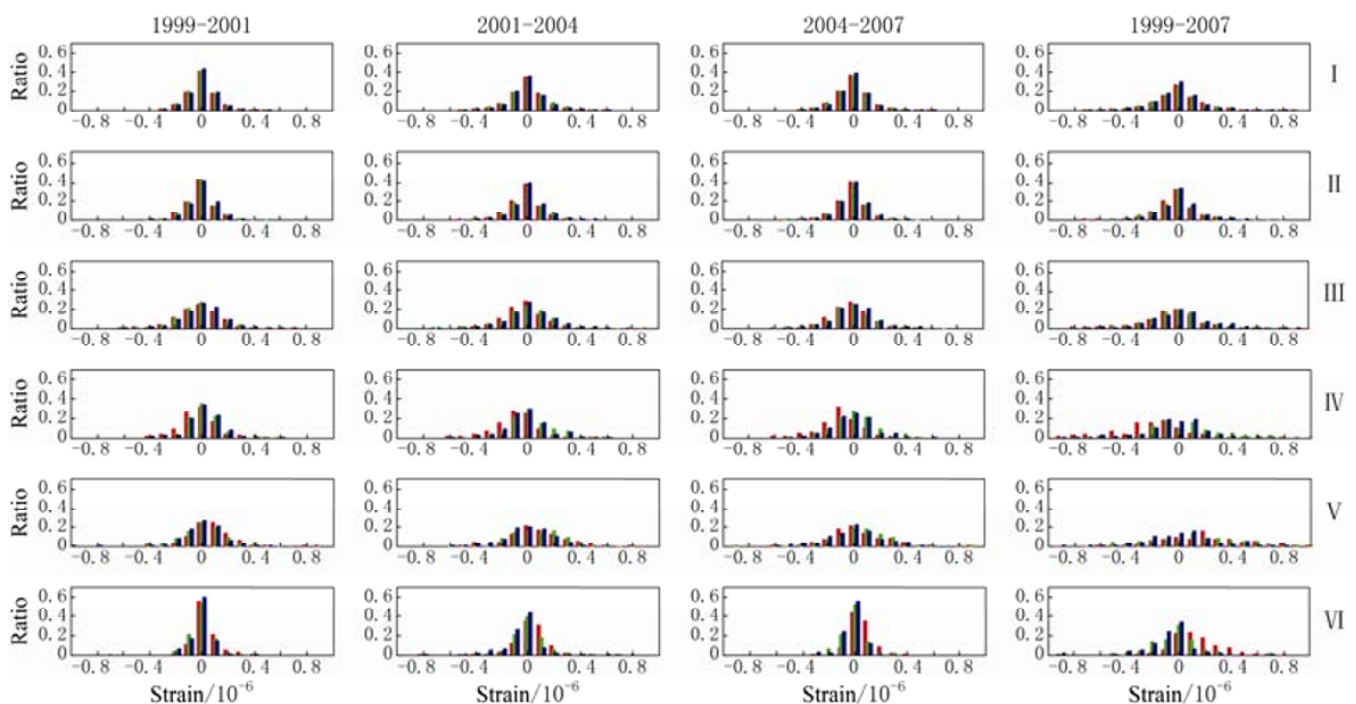

Figure 6 Distribution of accumulations of the first shear strain (red), the second shear strain (green) and the areal dilatation (blue) in ascending order in the four periods from 1999 to 2007 in the whole network (I), East China (II), North China (III), the Wenchuan earthquake area (IV), the Yunnan area (V) and West China (VI). 
1999 to 2007 (Figure 6). For example, the total numbers of triangles during the four periods in Yunnan are 196, 188, 180 and 182; while in the Wenchuan earthquake area the numbers are 223, 220, 217 and 217. Statistics in the same way for the whole network, North China and the east part of Chinese mainland shown in Figure 6 indicates that, for the same region, the same period and the same statistic interval, the numbers of the first shear strain, the second shear strain and areal dilatation, that is, the numbers of triangles, are almost the same even though the statistic patterns are different for different regions. The same statistics (Figure 6) made for the whole network, North China and East China shows that the numbers of the estimated first and second shear strains, areal dilatation and maximum shear strain were the same in general in the same interval and in the same region. Figure 6 indicates that in the Wenchuan earthquake area the number of negative first shear strains was significantly larger than the number of the negative second shear strains and also larger than the number of the negative areal dilatation, which is in well agreement with the result shown in Figure 2. But for West China the result is opposite: the number of positive first shear strains was significantly larger than the number of the positive second shear strains and also larger than the number of the positive areal dilatation. The opposite results for the Wenchuan earthquake area and West China may indicate certain mutual relationship between occurrences of the western Kunlun mountain pass earthquake and the Wenchuan earthquake. In Figure 6 the normal distribution of the strain components in Yunnan are flatter and the levels of average strains are higher than in other regions, but the systematic spatial trends of the strain components are less significant than that in the Wenchuan earthquake area.

Besides the results from the basic network ( $\mathrm{Gu}$ and Wang, 2006), the strain estimation from the regional network from 1999 to 2001 also shows similar statistic results of strain accumulations before the western Kunlun mountain pass earthquake in 2001, suggesting that statistic method is also useful in identifying anomalous strain accumulation areas before large earthquakes.

\section{Discussion}

The maps of horizontal displacements and strain accumulation are the fundamental "cloud images" for earthquake prediction. Though the results in this paper were obtained after the earthquake because of the data availability, the revealed characteristics of the horizontal crustal movements before the Wenchuan earthquake may be meaningful for studies on geodynamics, earthquake preparation process, earthquake monitoring and prediction.

The results of horizontal displacements obtained from the regional network show that the main driving tectonic force of the Wenchuan earthquake mainly come from the northward pushing of the Indian plate, added at the same time by the pushing of plates on the east and south. This result is in good agreement of the results of the fiducial stations of continuous GPS observations ( $\mathrm{Gu}$ and Wang, 2009). In comparison to the displacements in other regions, the most striking feature of the horizontal displacement near and around the earthquake area is the diverging eastward displacements, that is, the stations in the north of the epicenter moved in the ENE direction; while those in the south of epicenter moved in ESE direction and at stations near the epicenter displacements were smaller.

In order to obtain the anomalous information before the earthquake, the methods of both best fits by trend surfaces and statistics are used in the study on the future epicentral area from the strain accumulation in the regional network. Besides the epicentral area of the western Kunlun mountain pass earthquake of M8.1 in 2001 , the results of best fits by trend surfaces of the strain accumulation from 1999 to 2007 in regional network before the Wenchuan earthquake show that the event occurred at the eastern fringe of a large area with relatively large accumulation of the first shear strain and at the northeastern fringe of a smaller area with significant areal compression accumulation. On the west side of the Wenchuan earthquake, the accumulated first shear strain was left lateral shear strain in $\mathrm{N} 45^{\circ} \mathrm{E}$ direction with the largest fitted strain accumulation about $-4.0 \times 10^{-7}$. One belt of the anomalous shear strains was in general along the Xianshuihe fault, and the other belt was in general parallel to the earthquake fault. The Wenchuan earthquake occurred at the northeastern fringe of the area with relatively large accumulated compression within the anomalous area in Figure 2 with the largest fitted compression accumulations about $-3.5 \times 10^{-7}$.

Comparison of the distribution of seismic activities and the strain accumulation shows that both of them are of complicated stochastic features and governed by similar stochastic statistics. Obviously their complicatedness is determined by the complicated tectonics. Statistics of the strain accumulation for different periods and different regions demonstrates that anomalies, espe- 
cially those of the first shear strains, also appeared before the Wenchuan earthquake obtained by trend surface fittings. The results of the strain estimations in the regional network from 1999 to 2001 show similar anomalies of strain accumulation in West China before the 2001 western Kunlun mountain pass earthquake. Analyses on the strain accumulation before these two earthquakes suggest that statistic method may be useful in identifying the area with anomalous strain accumulations and the statistic results are also the most objective results from the observation data.

Comparison of the horizontal displacements and stain accumulation shows that the horizontal displacements are characterized by their significant group features on blocks and even their simultaneity ( $\mathrm{Gu}$ and Wang, 2009). The time series of horizontal displacements may be useful to analyze the development of the anomalous crustal movements, while the analyses on the accumulation of strain components may be useful to identify the spatial distribution of the anomalies.

Results of data processing at the data center of CMONOC were used for further processing and analyses in this paper.

Acknowledgments This paper is supported by National Key Technology Research and Development Program (2006BAC01B02-02-05) and Basic Research
Plan of the Institute of Earthquake Science, China Earthquake Administration (0207690239). Also GMT mapping tool developed by Paul Wessel from the University of Hawaii and Walter Smith from National Oceanic and Atmospheric Administration is used in this study.

\section{References}

Blewitt G (2007). Overview of the SNARF Working Group, its activities, and accomplishments. 8th SNARF Workshop AGU Fall Meeting. San Francisco, December 11.

Dong D, Fang P, Bock Y, Cheng M K and Miyazaki S (2002). Anatomy of apparent seasonal variations from GPS derived site position time series. $J$ Geophys Res 107(B4): ETG9-1-ETG9-16.

Gu G H (2006). Reference frame, coordinate transformations and crustal movements. Bulletin of Surveying and Mapping 8: 24-28 (in Chinese).

$\mathrm{Gu}$ G H (2007). Recent progress in researches on crustal movements through GNSS (GPS) observations. Recent Development in World Seismology (7): 9-15 (in Chinese).

Gu G H, Fu Y and Wang W X (2004). Horizontal crustal movement in Chinese mainland from 1999 to 2001. Acta Seismologica Sinica 17(Suppl.): 53-60.

Gu G H, Shen X H, Wang M, Zheng G M, Fang Y and Li P (2001). General characteristics of the recent horizontal crustal movement in Chinese mainland. Acta Seismologica Sinica 14(4): 384-393.

Gu G H, Sun H R, Sun H J and Yang C H (1998). Deduction of strain from GPS crustal deformation data in geodetic coordinate system. Crustal Deformation and Earthquake 18(3): 26-31 (in Chinese).

Gu G H and Wang L F (2006). Strain accumulation in Chinese mainland during 1998 to 2003 obtained by GPS observations. Earthquake 26(3): 1-8 (in Chinese with English abstract).

Gu G H and Wang W X (2009). Coseismic displacements of the great Wenchuan earthquakes in Sichuan detected by GPS observations. Earthquake 29(1): 92-99 (in Chinese with English abstract). 